\title{
Das TV-Festival als Bühne des Protests UND DER INNOVATION. DiE BRASILIANISCHEN MUSIKFESTIVALS 1965-1972
}

\author{
Carsten Heinke
}

\section{Brasilianische populäre Musik und Wettbewerb}

Die Idee des musikalischen Wettstreits ist in der Kultur Brasiliens erstaunlich präsent. Beispielsweise versuchen beim im Nordosten Brasiliens beheimateten cantoria zwei Sänger sich in ihrem Gitarrenspiel und vor allem in der Kunst ihrer improvisierten Verse gegenseitig zu übertrumpfen (Travassos 2000). Noch klarer sticht der Wettbewerbsgedanke beim Karneval in Rio hervor und dies gleich in mehrfacher Hinsicht. Die Sambaschulen wählen jedes Jahr in einem langen Prozedere aus einer großen Anzahl eingereichter Beiträge ihr Titelstück aus, das sie dann an den Karnevalstagen öffentlich präsentieren. Auch die beteiligten Instrumentalisten stehen in Konkurrenz zueinander, denn für die große Parade werden allein die ausgewählt, die rhythmisch sicher sind und weniger stark alkoholisiert scheinen. ${ }^{1}$ Vor allem aber ist der Karneval selbst ein Wettkampf, der zwischen den Sambaschulen ausgetragen wird. Hier gilt wie beim brasilianischen Fußball, dass schönes Spielen nicht unbedingt Nebensache ist. Was jedoch zählt, ist in erster Linie der Sieg.

Wettbewerbe innerhalb der brasilianischen Musik nutzten schon früh die Möglichkeiten der medialen Verbreitung, zunächst des aufkommenden Massenmediums Radio. Die Wahl einer Radiokönigin (Rainha do Rádio) unter jungen Sängerinnen, die 1948 das erste Mal stattfand, führte 1949 zu ausschreitungsartigen Zuständen zwischen den Fanclubs der beteiligten Interpretinnen, nachdem ein Getränkehersteller in einer Werbekampagne eine der Kandidatinnen protegiert hatte und ihr so zum Sieg verhalf (McCann

1 Vgl. die Dokumentation In The Heart Of Rio's Baterias (DVD, Le salon de musique 2002) von Philippe Nasse u.a., die eben diesen Auswahlprozess zeigt. 
2004: 203). Die Rolle der Radiowettbewerbe übernahmen ab den 1960er Jahren die TV-Festivals, sowohl hinsichtlich ihrer Funktion, d.h. der Auswahl des musikalischen Nachwuchses, als auch der durch sie hervorgerufenen Mobilisierung der Rezipienten. Insgesamt haben die TV-Festivals die Form von Wettbewerben in Brasilien neu definiert und geprägt.

Die Bezeichnung »Festival« lässt dabei zunächst nicht unbedingt auf einen Wettbewerb schließen, denn ebenso oft steht dieser Begriff auch für die bloße Präsentation künstlerischer Darbietungen, ohne Rivalität und anschließendes Küren eines Siegers. Wenn heutzutage in Brasilien allerdings in bewundernder und manchmal verklärender Weise von den Jahren der groBen Festivals als der Era dos Festivais gesprochen wird, bezieht sich dies ausschließlich auf die im Fernsehen ausgestrahlten musikalischen Wettbewerbe zwischen 1965 und 1972. ${ }^{2}$ Aus heutiger Sicht stellen diese Festivals ein Phänomen dar, denn von wenigen Ausnahmen abgesehen, sind seitdem nahezu alle bedeutenden Interpreten und Komponisten von brasilianischer populärer Musik, unter innen die »monstros sagrados« (heiligen Ungetüme, Napolitano 2002: 11) Chico Buarque, Elis Regina, Caetano Veloso und Milton Nascimento, durch ihre Teilnahme an einem Festival bekannt geworden.

Die Ansprüche, die an die brasilianischen Festivals zwischen 1965 und 1972 gestellt wurden, unterschieden sich deutlich von denen europäischer Wettbewerbe wie dem Grand Prix d'Eurovision. Ihre Relevanz lag einerseits in ihrer gesellschaftlichen Bedeutung als Widerstand gegen die herrschende Militärregierung und andererseits in der Suche nach neuen ästhetischen Wegen jenseits des etablierten Stils Bossa Nova, dem gemeinschaftlichen Generieren der Musikgenres MPB (Música Popular Brasileira), Canção de Protesto und Tropicalismo.

\section{Musikalische Entwicklung nach Bossa Nova und die Rolle des Fernsehens}

Um die Bedeutung der Festivals im Kontext der populären Musik verstehen zu können, soll zunächst auf die musikalische Entwicklung zwischen der nachlassenden Dominanz der Bossa-Nova-Bewegung ab 1962 und dem 1. Fes-

2 Zuza Homem de Mello (2003) bezieht den Wettbewerb des Senders TV Record von 1960, im Gegensatz zu vielen anderen Autoren, in seine historische Darstellung explizit mit ein. Der Wettbewerb von 1960 trug allerdings die Bezeichnung Festa und nicht Festival. Zudem hatte er keine große musikalische, gesellschaftliche oder kommerzielle Bedeutung und fällt aus der Kontinuität der Festivals zwischen 1965 und 1972 heraus. 
tival Nacional de Música Popular Brasileira 1965 eingegangen werden. Die chromatische Harmonik der Bossa Nova, die Nähe zum Jazz und die intime Vortragsweise ohne die Exaltiertheit der großen Samba-Sängerinnen und Sänger waren im Brasilien der späten 1950er Jahre eine musikalische Revolution, ihre Popularität im Ausland - und hier vor allem in den USA - ein kultureller Erfolg, der stark mit der Aufbruchsstimmung unter Präsident Juscelino Kubitschek und dem Bau der neuen Hauptstadt Brasilia assoziiert wurde. Spätestens seit dem Militärputsch 1964 wurde die Ästhetik der Bossa Nova jedoch als nicht mehr zeitgemäß bzw. als falsche Antwort auf die schwierige politische Lage und somit von der Wirklichkeit »entfremdet « (alienado) angesehen. Dies betraf vor allem ihre Texte um Liebe und Glückseligkeit in der Zweierbeziehung, aber auch ihre musikalische Struktur und intime Vortragsweise (Naves 2001: 26).

Kritik an der Bossa-Nova-Bewegung kam bereits 1962 aus deren Mitte heraus von Carlos Lyra, der sowohl den musikalischen Einfluss des Jazz als auch ihre politische Unbekümmertheit ablehnte und zum Vorreiter einer »engagierten« (engajado) populären Musikbewegung in Brasilien wurde. Von da an richtete sich die Betonung von Authentizität und der Rückgriff auf traditionelle Formen einerseits bewusst gegen die Kultiviertheit der >amerikanisierten Bossa-Nova-Bewegung und sollte andererseits eine Verbindung zum povo, d.h. zu den Fabrikarbeitern und Landbewohnern schaffen, was in der Charakterisierung der neuen Musik als »nacional-popular« zum Ausdruck kam und im Kontext der Festivals dann zum Genre Canção de Protesto (Protestlied) führte (Treece 1997: 4).

Eine Abkehr von der Bossa-Nova-Bewegung war die Musik der Jovem Guarda, die wegen ihrer vermeintlichen Nähe zu den Beatles auch lê-iê-iê genannt wurde und in Roberto Carlos und Erasmo Carlos ihre populärsten Vertreter hatte. Nicht zuletzt wegen des großen kommerziellen Erfolges wurde die Musik der Jovem Guarda von den Intellektuellen kritisch beäugt und als rein kommerzielles Phänomen abgetan. ${ }^{3}$ In ideologischer Hinsicht galt ihnen diese Musik als entfremdet und konnte ihrem Anspruch an Musik als Ausdruck von Authentizität nicht gerecht werden. Zeitgleich zu dieser Entwicklung blühte eine Variante der Bossa Nova in einigen Clubs der Cobacabana erneut auf. Der Name der kleinen Gasse Beco das Garrafas, wo Shows von Elis Regina, Wilson Simonal oder Lennie Dale stattfanden, wurde zum Synonym für eine Musik, die Elemente der Bossa Nova zwar noch in sich trägt, bisweilen jedoch stark nach einer brasilianischen Variante des Rat

3 Bei Claus Schreiner (1977: 187) heißt es: »Denen die Lieder der kritischen engagierten Musiker zu anspruchsvoll waren, bot die Platten-Industrie eine Alternative.« 
Pack klingt. Zwar galt dieses Genre ebenso wie die Bossa-Bewegung und die Jovem Guarda zunächst als politisch entfremdet, gerade Elis Regina stellte aber bald als Interpretin kritischer Musiktitel einen wichtigen Teil der engagierten Bewegung dar.

Bei allen Differenzen hatten die konkurrierenden Musikstile Jovem Guarda und Beco das Garrafas die Gemeinsamkeit, über eigens für sie eingerichtete Fernsehsendungen verfügen zu können. Schon die Bossa Nova hatte ja zumindest im Ausland einen Großteil ihrer Popularität dem visuellen Medium Film zu verdanken. Marcel Camus' Orfeu Negro von 1959 fand in Brasilien selbst jedoch wenig Beachtung und spielte dort hinsichtlich der Rezeption des Genres keine Rolle (Perrone 2002). Erst in den Folgejahren, besonders zwischen 1965 und 1969, war die Verbreitung von populärer Musik an bewegte Bilder gekoppelt, nun aber im Medium Fernsehen.

Die höchsten Zuschauerzahlen hatte dabei das von Elis Regina präsentierte Programm O Fino da Bossa (ab Mai 1965 ausgestrahlt), das neben den Festivals zur Plattform für politisch kritische Musiker avancierte, und die Sendung Jovem Guarda (ab September 1965). Die beiden Formate unterschieden sich nicht bloß hinsichtlich der präsentierten Musik, vielmehr wurden zwei unterschiedliche ideologische Richtungen mit ihnen assoziiert, die TV Record geschickt zu vermarkten wusste: die engagierte, nationalpopuläre von O Fino da Bossa im Gegensatz zu der (vermeintlich) kommerziellen und international ausgerichteten von Jovem Guarda. Dieser Konflikt blieb als Wesensmerkmal bei der Wahrnehmung von populärer Musik in der zweiten Hälfte der 1960er Jahre bestimmend und erreichte mit den Festivals seinen Höhepunkt.

Neben der großen Anzahl von Programmen fällt vor allem die Bündelung im Sender TV Record auf, der mit Ausnahme des Tropicalismo Sendungen aller Genres beheimatete (vgl. Tab. 1). Insofern muss Caetano Veloso zugestimmt werden: »TV Record specialized in music as did no other network in the world, as far as I know, before the advent of $M T V \ll$ (Veloso 2002: 111). Auch als Organisator von Festivals zeichnete TV Record neben den Sendern TV Globo und TV Excelsior verantwortlich.

Bei aller Plausibilität der dargestellten musikalischen Entwicklungen bleiben zwei Dinge zu beachten: erstens bedeutete der Beginn der neuen Strömungen nicht zugleich das Ende der ursprünglichen Bossa Nova; ${ }^{4}$ zweitens betraf diese Entwicklung nur einen kleinen Ausschnitt der Bevölkerung, in der Hauptsache die weiße, universitär gebildete Mittelschicht. Für viele

4 Erst 1963 erscheint »Garota de Ipanema« von Tom Jobim und Vinicius de Moraes, später die ebenfalls von Jobim komponierten Stücke »Wave« (1967) und »Aguas de Março« (1972). 
andere blieb der Samba der musikalische Bezugspunkt und die beschriebene Entwicklung somit weitgehend bedeutungslos. Wichtig für die nichtprivilegierte Gesellschaftsschicht wurde zudem die Verbreitung des Forró, mit der Einrichtung von Tanzhäusern (gafieiras) der Zuwanderer aus dem Nordosten in der zweiten Hälfte der 1960er Jahre in Rio und ab 1962 in São Paulo (Pinto 1995), sowie das schlagerähnliche Genre Brega (Araújo 2002).

\begin{tabular}{|c|c|c|c|c|}
\hline $\begin{array}{l}\text { Gesellschaft- } \\
\text { liche Position } \\
\text { laut Bildungs- } \\
\text { schicht }\end{array}$ & Genre & Besetzung & \begin{tabular}{|l|} 
Wichtige \\
Vertreter
\end{tabular} & TV-Sendung \\
\hline \multirow[t]{3}{*}{ engajado } & Samba & $\begin{array}{l}\text { Kleines } \\
\text { Ensemble oder } \\
\text { große escola de } \\
\text { samba }\end{array}$ & $\begin{array}{l}\text { Paulinho da Viola, } \\
\text { Clara Nunes, } \\
\text { Elizeth Cardoso... }\end{array}$ & $\begin{array}{l}\text { Bossaudade } \\
\text { (TV Record) }\end{array}$ \\
\hline & $\begin{array}{l}\text { MPB (Música } \\
\text { Popular } \\
\text { Brasileira) }\end{array}$ & $\begin{array}{l}\text { verschieden, } \\
\text { ausladender } \\
\text { Stil, keine } \\
\text { E-Gitarren }\end{array}$ & $\begin{array}{l}\text { Chico Buarque, } \\
\text { Edu Lobo, } \\
\text { Nara Leão... }\end{array}$ & $\begin{array}{l}\text { Pra Ver a Banda } \\
\text { passar } \\
\text { (TV Record) }\end{array}$ \\
\hline & $\begin{array}{l}\text { Canção de } \\
\text { Protesto }\end{array}$ & $\begin{array}{l}\text { verschieden, } \\
\text { teilweise nur } \\
\text { Sänger mit } \\
\text { (akustischer) } \\
\text { Gitarre } \\
\end{array}$ & $\begin{array}{l}\text { Geraldo Vandré, } \\
\text { Sidney Miller, } \\
\text { Sérgio Ricardo }\end{array}$ & $\begin{array}{l}\text { Disparada } \\
\text { (TV Record), } \\
\text { Caminhando } \\
\text { (TV Globo) }\end{array}$ \\
\hline $\begin{array}{l}\text { alienado/ } \\
\text { engajado }\end{array}$ & \begin{tabular}{|l} 
Beco das \\
Garrafas
\end{tabular} & \begin{tabular}{|l} 
Tendenz zu \\
größeren \\
Besetzungen
\end{tabular} & $\begin{array}{l}\text { Elis Regina, } \\
\text { Wilson Simonal, } \\
\text { Jorge Ben... }\end{array}$ & $\begin{array}{l}\text { O fino da Bossa } \\
\text { (TV Record) } \\
\text { Frente Única } \\
\text { (TV Record) }\end{array}$ \\
\hline alienado & Jovem Guarda & Rockband & $\begin{array}{l}\text { Roberto Carlos, } \\
\text { Erasmo Carlos, } \\
\text { Wanderléa... }\end{array}$ & $\begin{array}{l}\text { Jovem Guarda } \\
\text { (TV Record) }\end{array}$ \\
\hline $\begin{array}{l}\text { verhandelnd } \\
\text { zwischen } \\
\text { engajado und } \\
\text { alienado }\end{array}$ & $\begin{array}{l}\text { Tropicalismo } \\
\text { (ab 1967) }\end{array}$ & $\begin{array}{l}\text { Rockband, } \\
\text { teilweise mit } \\
\text { Orchester und } \\
\text { traditionellen } \\
\text { Instrumenten } \\
\text { (plus Studio- } \\
\text { effekten) } \\
\end{array}$ & $\begin{array}{l}\text { Caetano Veloso, } \\
\text { Gilberto Gil, } \\
\text { Gal Costa, } \\
\text { Os Mutantes, } \\
\text { Tom Zé, } \\
\text { Nara Leão... }\end{array}$ & \begin{tabular}{|l|} 
Divino, \\
maravilhoso \\
(TV Tupi)
\end{tabular} \\
\hline
\end{tabular}

Tabelle 1: Musikgenres der zweiten Hälfte der 1960er Jahre in Brasilien und ihre TV-Sendungen ${ }^{5}$

5 Die Tabelle dient einem Überblick. Sie deutet die vielfachen Querverbindungen zwischen den Musikarten an, deckt aber nicht alle Musikrichtungen der Zeit ab. Die Afro-Sambas von Baden Powell und Vinicius de Moraes, die hier wegen der Schwierigkeit einer exakten Zuordnung fehlen, greifen zum Beispiel ähnlich wie die Stücke von Edu Lobo auf regionale musikalische Traditionen zurück, ohne jedoch in ähnlicher Weise einen explizit ideologischen Hintergrund zu bemühen. Auch ihre Popularität basiert auf der Fernsehsendung $O$ fino da Bossa. Zu den ab 1965 sich im Rahmen der Festivals konstituierenden Genres MPB, Canção de Protesto und Tropicalismo vgl. die Darstellung weiter unten. 


\section{Die Musikshow als kultureller Protest}

Nach einer kurzen Phase der Hoffnung auf wirtschaftlichen Anschluss an die westlichen Industrienationen und einem dadurch erhöhten Lebensstandard kam es nach einer krisenhaften Wirtschaftsentwicklung und politischen Instabilität 1964 in Brasilien zum Putsch durch das Militär. Die Militärdiktatur, die bis 1985 andauerte, brachte zwar einen wirtschaftlichen Aufschwung, führte andererseits jedoch zum Verlust von Grundrechten wie dem der freien Meinungsäußerung und der politischen Wahlmöglichkeit.

Erstaunlicherweise betrafen die Einschränkungen in der Anfangszeit nach dem Putsch kaum die populäre Musik, da ihr vom Staat wenig Aufmerksamkeit geschenkt wurde (Zan 2003: 213). Ohnehin pflegte die Militärregierung nach außen eine demokratische Fassade und hatte auch kein Interesse daran, die Entwicklung des lukrativen Schallplattenmarktes zu bremsen. Ihre Aktionen konzentrierten sich zunächst auf die oppositionellen politischen Parteien. Nachdem diese aufgelöst worden waren, folgte die Neugründung der alleinigen Oppositionspartei MDB (Movimento Democrático Brasileiro), die in ihrem Handlungsspielraum vom Gutdünken der Militärs abhängig blieb (vgl. Zoller 2000). Da das Parlament damit zur Bedeutungslosigkeit degradiert wurde und auch die studentischen Organisationen nicht mehr operieren durften, blieb als wahrnehmbare Form von Widerstand nur der kulturelle Protest.

Die politische Revue Opinão, die im Dezember 1964 in Rio de Janeiro uraufgeführt wurde, gilt heute als erste wichtige Reaktion auf die Militärregierung aus dem Bereich der populären Musik. In einer Mischung aus Anekdoten, Dialogen und Musikstücken, die u.a. den harten Überlebenskampf der Landbevölkerung im Nordosten - der bis heute ärmsten Region Brasiliens - thematisierten, wurde gegen das Regime Stellung bezogen (vgl. Napolitano 2004: 50). Trotz des großen Erfolgs der Revue griff die Militärregierung nicht ein, wohl weil die Reichweite einer Theateraufführung lokal und gesellschaftlich sehr begrenzt blieb. Der Einfluss beschränkte sich im Wesentlichen auf die Studierenden, für deren Musikpräferenz die ideologische Einstellung eine wesentliche Rolle spielte. In ihrer Mehrheit waren sie politisch linksgerichtet, was jedoch eine nationale Haltung mit einschloss. Demnach war es Hauptaufgabe von Künstlern und Intellektuellen, an das politisch revolutionäre Bewusstsein der Leute zu appellieren, indem kulturelle Symbole der Landbevölkerung Brasiliens zum Zweck eines anvisierten Bündnisses aufgegriffen werden sollten. Aus diesem Grund war innerhalb der populären Musik jeglicher anglo-amerikanische Einfluss, vor allem aber 
die Verwendung von E-Gitarren, egal in welchem Kontext, verpönt und galt als Kommerzialisierung. Ausdruck hierfür war der Protestmarsch Frente Única »gegen die E-Gitarre« am 17. Juli 1967 in São Paulo (vgl. Mello 2003: 181). Ideologische Motive dürften zwar bei den Beteiligten dieser skurrilen Veranstaltung ausschlaggebend gewesen sein, dem Veranstalter des Marsches, TV Record, ging es allerdings primär um eine wirkungsvolle Werbung für die neue Fernsehsendung Frente Única da MPB (Villaça 2004: 75). Dennoch stellt diese Episode den Hintergrund für den bei Musikern und Fans sich verschärfenden Konflikt zwischen den engagierten nationalen Musikrichtungen (MPB/Canção de Protesto) und den die internationale Rockmusik einbeziehenden Genres Jovem Guarda bzw. Tropicalismo auf den Festivals 1967/68 dar. Diese Festivals boten einen Raum für diese Auseinandersetzung, ebenso aber auch die Möglichkeit, dem Frust gegen das Regime freien Lauf zu lassen:

»The festivals afforded a rare opportunity for disaffected youth - leftist or otherwise - to register its frustrations at the establishment, and by extension the military regime, in a form of cultural contestação [Protest; Hervorhebung im Original, C.H.] despite the fact that television itself remained an elitecontrolled medium« (Stroud 2000: 98).

Neben seiner relativierenden Beurteilung des politisch linken Bewusstseins der studentischen Zuhörer macht Sean Stroud auch auf das grundsätzliche Paradox der Festivals aufmerksam: der problematischen Vereinbarkeit von politischem Widerstand mit dem, wenn auch nicht staatseigenen, so doch regierungsnahen Medium Fernsehen. Tatsächlich wurde der Widerspruch zwischen der Ausstrahlung in einem Massenmedium, dessen Betreiber vor allem kommerzielle Interessen verfolgen, und dem von Publikum und Presse an MPB/Canção de Protesto gestellten Anspruch auf Authentizität wenig problematisiert. Deren Musikstücke waren aber immer auch Produkte der Fernseh- und Phonoindustrie, die marktorientiert und gewinnbringend lanciert wurden. Diese Dimension wurde von vielen Musikern ausgeblendet. Ihnen galt das Erreichen der unteren Bevölkerungsschichten als primäres Ziel, um auf die Möglichkeit von politischer Einflussnahme aufmerksam zu machen. Der Fernsehkonsum war allerdings eine Angelegenheit, von der nichtprivilegierte Bevölkerungsteile Brasiliens größtenteils ausgeschlossen waren, bedenkt man, dass in einer Stadt wie São Paulo die - verglichen mit heutigen europäischen Maßstäben - prozentual relativ kleine Mittelschicht über 70\% der Fernsehgeräte verfügte (Napolitano 2004: 58). Hieraus resultierte der Vorwurf an die Musiker des Canção de Protesto, ihre Lieder 
würden die adressierten Gesellschaftsschichten nicht einmal erreichen (vgl. Villaça 2004: 72).

\section{Organisation und Ablauf der Festivals}

Das 1. Festival Nacional de Música Popular Brasileira wurde 1965 von TV Excelsior veranstaltet. Der Sender stellte die Ausstrahlung von Festivals jedoch schon im Folgejahr ein, nachdem der Fernsehproduzent Solano Ribeiro den Sender verlassen hatte. Ribeiro wechselte zu TV Record und leitete von 1966 an das jährlich stattfindende Festival de Música Popular Brasileira. Als festes Schema der Festivals von TV Record setzte sich ein Ablauf mit drei Vorrunden und einer Finalveranstaltung durch, ausgeführt in einer Zeitspanne von zwei bis drei Wochen.

Ebenfalls von 1966 an fand jährlich der Wettbewerb FIC - Festival Internacional da Cancão Popular (Internationales Festival des populären Liedes) statt, der vom neugegründeten Sender Globo ausgestrahlt wurde. Im Unterschied zu den zuvor genannten Festivals wurde die Veranstaltung bewusst auf internationale Interpreten und Komponisten ausgeweitet. Sie war geteilt in einen nationalen Vorentscheid (zwei Vorrunden plus Finale) und den anschließenden internationalen Wettbewerb, dem die brasilianische Öffentlichkeit jedoch deutlich weniger Aufmerksamkeit schenkte. ${ }^{6}$

Als Inspirationsquelle und Vorlage für die Organisation der Festivals diente das seit 1951 stattfindende Festival Sanremo. Der Grand Prix d'Eurovision ist dagegen in Brasilien bis heute offensichtlich selbst >Festivalexperten< unbekannt. 1965 ließ sich Solano Ribeiro das Reglement von Sanremo schicken, um es, wie er selbst schreibt, weitgehend identisch in Brasilien umzusetzen (Ribeiro 2002: 67). Wie das Festival Sanremo waren auch die brasilianischen Festivals Kompositionswettbewerbe. Bedingung für eine Anmeldung war das Einreichen einer Partitur, erst bei späteren Festivals wurden auch Aufnahmen auf Tonträgern akzeptiert (Ribeiro 2002: 68). Die

6 Laut Zuza Homem de Mello war diese Ablehnung mehr oder weniger gerechtfertigt, denn der Gewinner des 1. FIC war Helmut Zacharias mit dem »abgeschmackten« (»insípida«, Mello 2003: 169) Titel »Frag den Wind « in der Interpretation von Inge Brück. Deutschsprachige Teilnehmer am FIC waren außerdem die Sängerin Alexandra (»llusionen«) und Udo Jürgens (»Geh' vorbei«). Es ist auffallend, dass Mello dem internationalen Teil in seiner minutiösen Darstellung A Era dos Festivais im Textteil kaum, in der tabellarischen Auflistung der Daten zu den Festivals dann überhaupt keine Aufmerksamkeit mehr schenkt. Bedeutung erhielt der internationale Teil des FIC rückblickend, weil Gilberto Gil dort den Jamaikaner Jimmy Cliff kennen lernte, was die Initialzündung für die brasilianische Reggaebewegung bedeutete (vgl. Godi 2002: 215). 
Bewertung der Musiktitel oblag zwei streng getrennten Jurys aus Fachleuten. Die Vorauswahl der eingegangenen Partituren und die Entscheidung zur Zulassung zum Wettbewerb traf eine Kommission von wenigen Personen: im Falle des Festivals im Jahr 1965 der Lyriker Augusto de Campos ${ }^{7}$, der Semiotikprofessor Décio Pignatari, der Dirigent Damiano Cozzella, der Komponist Caetano Zammataro und der Musiker Amilton Godoy (Ribeiro 2002: 68). Die Jury des Wettbewerbs selbst bestand aus einer etwas größeren Personengruppe, die sich aus Journalisten, Kritikern, Dirigenten und einem Produzenten zusammensetzte. Aufschlussreich ist der große Anteil von Personen aus der E-Musik: beim Festival des Senders TV Record 1967 war dies z. B. der bekannte Pianist und Bachinterpret João Carlos Martins. Die erlesenen Namen sorgten für ein elitäres Flair, sollten aber auch eine hohe Fachkompetenz unterstreichen. So ließ sich rechtfertigen, dass der Jury keine Vorschriften in der Beurteilung der Musiktitel gemacht wurden und es auch keine festgelegten Bewertungskriterien gab (vgl. Stroud 2000: 99).

Eine Besonderheit stellt das 4. Festival da Música Popular Brasileira 1968 dar, bei dem neben der Fachjury auch eine so genannte júry popular zugelassen wurde. Die Initiative ging von Komponisten aus, die sich nicht dem Gutdünken einer kleinen Expertengruppe aussetzen wollten (Mello 2003: 308). Neben der offiziellen Jury, bestehend aus je sieben Mitgliedern in Rio und São Paulo, gehörten 98 Nichtfachleute aus dem Inland der júry popular an, darunter viele Sportler. Der angestrebte Versuch von mehr Volksnähe bei der Bewertung wurde dadurch eingeschränkt, dass diese Jury auf die Auswahl der zur Vorrunde zugelassenen 36 Titel keinen Einfluss hatte und nur das Weiterkommen in die Endrunde mitbestimmen konnte. Dort wurden die Ergebnisse der beiden Bewertungskommissionen unabhängig voneinander gewertet und prämiert, was de facto den Preis der júry popular zu einer Nebenerscheinung machte. Bemerkenswert ist die Divergenz in der Bewertung der beiden Jurys. Das experimentelle Stück »2001 « von Os Mutantes erhielt zum Beispiel im ersten Teil der Vorrunde unter 18 aufgeführten Titeln die höchste Punktzahl von den Experten und die geringste von der júry popular (Mello 2003: 316).

Während die Bewertung der Musik insgesamt in der Hand von Experten blieb, wurde sich das (Live-)Publikum seiner Macht der Beeinflussung doch immer mehr bewusst. Es lauschte den Darbietungen der Interpreten nicht in stiller Kontemplation, sondern bekundete Sympathien und Antipathien durch frenetischen Jubel bzw. Buhrufe (vaias). Gerade das lautstarke Aus-

7 Campos ist mit seinem Sammelband Balanço da Bossa e outras bossas (1993, E: 1978) zudem ein früher Vertreter dessen, was wir heute einen Poptheoretiker nennen. 
buhen wurde zum charakteristischen Merkmal der Festivals. Ganz offensichtlich hatte das Publikum genaue Vorstellungen von Qualität und Ansprüche an Inhalte, die bestimmte Musiker zu vertreten hatten. ${ }^{8}$ Auch Urteile der Jury wurden ausgebuht. Eine Folge der vaias waren die gesteigerte Aufmerksamkeit der Festivals in der Öffentlichkeit, aber auch ein Fernbleiben von etablierten Stars aus Angst vor einem Tumult im Publikum, was als Konsequenz Raum für eine neue Generation von Komponisten und Interpreten schuf (Mello 2003: 310). Die Beeinflussung der Jury wirkte sich am stärksten auf das Resultat des 2. Festivals da MPB 1966 aus, bei dem der erste Preis in einer Konzessionsentscheidung sowohl Chico Buarque als auch Geraldo Vandré/Théo de Barros zugesprochen wurde - aus Furcht vor einem Aufruhr der Zuschauer.

Die Ziele, die mit den Festivals verfolgt wurden, waren je nach Personenkreis unterschiedlich. Für den Initiator Solano Ribeiro war unabhängig vom Wettbewerbsgedanken entscheidend, dass die Festivals die musikalische Realität in Brasilien abbildeten. ${ }^{9}$ Für die Fernsehsender und Plattenfirmen standen kommerzielle Interessen im Vordergrund, immerhin waren die regelmäßig erscheinenden Zusammenstellungen der erfolgreichsten Festivalbeiträge ein bedeutendes Segment auf dem Schallplattenmarkt. ${ }^{10}$ Glaube und Anspruch von Jury, Musikern und Publikum war, das tatsächlich qualitativ hochwertigste Musikstück unabhängig von subjektivem Geschmack, Moden und kommerziellen Perspektiven auszuzeichnen, wobei die Vorstellung von Qualität mit der politischen Ausrichtung der Musikstücke verbunden war. Typisch für eine kritische Sicht auf die unterschiedliche Motivation zur Austragung dieser Wettbewerbe ist das Fazit von Ramon Casa Vilarino: »Die MPB-Festivals waren Rückzugsorte für Publikum, Komponisten und Interpreten und zugleich Ausgangspunkte für ihre Karrieren; für die Veranstalter waren es Schaufenster zur Ausstellung ihrer Ware. «" ${ }^{11}$

8 Sérgio Ricardo zerstörte beim Festival Record 1967 aus Wut über die lärmenden Zuschauer auf offener Bühne seine Gitarre, weil das Publikum sein Stück »Beto Bom de Bola«, in dem er als Protestsänger die Geschicke eines Fußballspielers thematisiert, ablehnte (Campos 1993: 129).

9 »Minha intenção sempre foi utilizar o festival como um painel do que estava sendo feito na música popular em tudo o país « (Ribeiro 2002: 95).

10 Die Singleauskopplungen erlangten nicht diesen Stellenwert. Ein >Klassiker < der Festivalära, Edo Lobos »Ponteio«, erreichte in der brasilianischen Hitparade nur den achten Platz (vgl. Napolitano 2001: 205).

11 »Os festivais de MPB eram locais de resistência para público, compositores e intérpretes e também espaço para iniçio da carreira destes; para os patrocinadores eram vitrinas em que exibiriam seus produtos« (Vilarino 2000: 96). 
DAs TV-Festival als BÜHNe des PROTESTS UND DER INNOVATION

\begin{tabular}{|c|c|c|c|c|}
\hline \multirow[t]{2}{*}{ Festival } & \multirow[t]{2}{*}{ Jahr } & \multicolumn{3}{|c|}{ Sieger } \\
\hline & & Titel & Interpret & Komponist \\
\hline $\begin{array}{l}\text { 1. Festival Nacional } \\
\text { de MPB } \\
\text { (TV Excelsior) }\end{array}$ & 1965 & »Arrastão《 & Elis Regina & $\begin{array}{l}\text { Edu Lobo/ } \\
\text { Vinicius de Moraes }\end{array}$ \\
\hline $\begin{array}{l}\text { 2. Festival Nacional } \\
\text { de MPB } \\
\text { (TV Excelsior) }\end{array}$ & 1966 & $\begin{array}{l}\text { »Porta } \\
\text { estandarte« }\end{array}$ & $\begin{array}{l}\text { Airto Moreira/ } \\
\text { Tuca }\end{array}$ & $\begin{array}{l}\text { Geraldo Vandré/ } \\
\text { Fernando Lona }\end{array}$ \\
\hline \multirow{2}{*}{$\begin{array}{l}\text { 2. Festival da MPB } \\
\text { (TV Record) }\end{array}$} & \multirow[t]{2}{*}{1966} & »A Banda« & Nara Leão & Chico Buarque \\
\hline & & »Disparada« & Jair Rodrigues & $\begin{array}{l}\text { Geraldo Vandré/ } \\
\text { Théo de Barros }\end{array}$ \\
\hline $\begin{array}{l}\text { 1. FIC - Festival } \\
\text { Internacional da } \\
\text { Cancão Popular } \\
\text { (TV Rio) }\end{array}$ & 1966 & »Saveiros« & Nana Caymmi & $\begin{array}{l}\text { Dori Caymmi und } \\
\text { Nelson Motta }\end{array}$ \\
\hline $\begin{array}{l}\text { 3. Festival da MPB } \\
\text { (TV Record) }\end{array}$ & 1967 & »Ponteio« & $\begin{array}{l}\text { Edu Lobo/ } \\
\text { Marília Medalha }\end{array}$ & $\begin{array}{l}\text { Edu Lobo/ } \\
\text { Capinan }\end{array}$ \\
\hline $\begin{array}{l}\text { 2. FIC } \\
\text { (TV Globo) }\end{array}$ & 1967 & »Margarida« & $\begin{array}{l}\text { Gutemberg } \\
\text { Guarabira/ } \\
\text { Grupo Manifesto }\end{array}$ & $\begin{array}{l}\text { Gutemberg } \\
\text { Guarabira }\end{array}$ \\
\hline $\begin{array}{l}\text { 1. Bienal do Samba } \\
\text { (TV Record) }\end{array}$ & 1968 & »Lapinha« & Elis Regina & $\begin{array}{l}\text { Baden Powell/ } \\
\text { Paulo César } \\
\text { Pinheiro }\end{array}$ \\
\hline $\begin{array}{l}\text { 3. FIC } \\
\text { (TV Globo) }\end{array}$ & 1968 & 》Sabiá« & Cynara/Cybele & $\begin{array}{l}\text { Tom Jobim/ } \\
\text { Chico Buarque }\end{array}$ \\
\hline \multirow[t]{2}{*}{$\begin{array}{l}\text { 4. Festival da MPB } \\
\text { (TV Record) }\end{array}$} & \multirow[t]{2}{*}{1968} & $\begin{array}{l}\text { »São, São } \\
\text { Paulo meu } \\
\text { amor« }\end{array}$ & $\begin{array}{l}\text { Tom Zé/ } \\
\text { Canto 4/ } \\
\text { Os Brasões }\end{array}$ & Tom Zé \\
\hline & & $\begin{array}{l}\text { júry popular: } \\
\text { »Benvinda« }\end{array}$ & $\begin{array}{l}\text { Chico Buarque/ } \\
\text { MPB } 4\end{array}$ & Chico Buarque \\
\hline $\begin{array}{l}\text { 4. FIC } \\
\text { (TV Globo) }\end{array}$ & 1969 & $\begin{array}{l}\text { »Cantiga por } \\
\text { Luciana« }\end{array}$ & Evinha & $\begin{array}{l}\text { Edmundo Souto/ } \\
\text { Paulinho Tapajós }\end{array}$ \\
\hline $\begin{array}{l}\text { 5. Festival da MPB } \\
\text { (TV Record) }\end{array}$ & 1969 & $\begin{array}{l}\text { "Sinal } \\
\text { Fechado« }\end{array}$ & Paulinho da Viola & Paulinho da Viola \\
\hline $\begin{array}{l}\text { 5. FIC } \\
\text { (TV Globo) }\end{array}$ & 1970 & $\gg B R-3 \ll$ & $\begin{array}{l}\text { Toni Tornado/ } \\
\text { Trio Ternura/ } \\
\text { Quartetto Osmar } \\
\text { Milito }\end{array}$ & $\begin{array}{l}\text { Antônio Adolfo/ } \\
\text { Tibério Gaspar }\end{array}$ \\
\hline $\begin{array}{l}\text { 6. FIC } \\
\text { (TV Globo) }\end{array}$ & 1971 & »Kyrie« & Trio Ternura & $\begin{array}{l}\text { Paulinho Soares/ } \\
\text { Marcelo Silva }\end{array}$ \\
\hline $\begin{array}{l}\text { 7. FIC } \\
\text { (TV Globo) }\end{array}$ & 1972 & $\begin{array}{l}\text { »Fio } \\
\text { Maravilha« }\end{array}$ & Maria Alcina & Jorge Ben \\
\hline
\end{tabular}

Tabelle 2: Die Festivals in zeitlicher Reihenfolge (vgl. Mello 2003: 438-463) 


\section{MPB und Música de Festival}

Ab 1965 wurden die TV-Festivals zum Sammelbecken neuer musikalischer Strömungen. Der Name Festivais da Música Popular Brasileira übertrug sich dabei als Kürzel MPB auf die gesamte, bei den Festivals präsentierte Musik, bezeichnete somit aber notwendigerweise eine Anzahl von »objetos híbridos« (Napolitano 2002: 2), d.h. eine Pluralität diverser Genres und Personalstile. Dennoch beinhaltete das Akronym MPB in strengem Sinne längst nicht alle populäre Musik aus Brasilien. Ausschlaggebend für die Zuordnung zur MPB war die Sorge der Musiker um die sozialen Verhältnisse in Brasilien, der Widerstand gegen die Militärregierung sowie ein hoher ästhetischer Wert der Musik selbst (Napolitano 2004: 57). Neben der positiven, oft euphorisch als Qualitätssiegel verwendeten Bezeichnung MPB besaß der zur damaligen Zeit von Chico Buarque geprägte Begriff Música de Festival eine negative Konnotation und verwies auf den uninspirierten Stil eines Großteils der Beiträge (Napolitano 2001: 217). Hier schwang die Befürchtung mit, der kreative Impuls der Festivals könne bereits verpufft und die Beiträge nur Plagiate von Altbekanntem sein.

Recht schnell kristallisierte sich ein spezifischer Typ von Festivalbeiträgen heraus, sowohl hinsichtlich der Musik, der Texte als auch der Performance. Musikalisch bildet Edu Lobos Stück »Ponteio« von 1967 eine Folie, an der sich spätere Festivalbeiträge orientierten. Es basiert auf einem stark synkopierten baião, einem Rhythmus aus dem brasilianischen Nordosten. Regionale Stile (samba, baião, moda de viola, marcha etc.) bildeten von da an die Grundlage eines Großteils der präsentierten Stücke. Außerdem galt der Verzicht auf elektronisches Instrumentarium als Selbstverständlichkeit. Aufschlussreich ist, dass selbst die Interpreten der Jovem Guarda, die von 1967 an bei den Festivals mit eigenen Stücken antraten, von ihrem ursprünglichen Stil abwichen, demonstrativ auf E-Gitarren verzichteten und sich so einer Folie von typischer brasilianischer Festivalmusik annäherten. Daneben setzte sich als weiteres stilprägendes Phänomen das Protestlied (Canção de Protesto) durch, prototypisch in Geraldo Vandrés Stück »Disparada« von 1966, das zwar ebenfalls auf einer regionalen Stilistik basiert, nämlich den improvisierten Gesängen aus dem Hinterland Brasiliens, bei denen allerdings fast vollständig auf Synkopierungen verzichtet wird. Chromatische Elemente in Harmonik und Melodik wurden zudem zugunsten der Kadenzharmonik aufgeben (vgl. Treece 1997: 25). Im Gegensatz zu der ideologischen und nur bedingt in der Musik selbst vollzogenen Abwendungen 
von der Bossa-Nova-Tradition bei Carlos Lyra betraf der Bruch bei Vandré damit praktisch alle musikalischen Parameter (Treece 1997: 5).

Der ideologischen Einstellung entsprechend und mit den musikalischen Elementen korrespondierend, behandelten die Texte der Festivalsongs die Lebensgewohnheiten der seinfachen Leute außerhalb der urbanen Metropolen. Ein Tabu war es allerdings, die gemeinsame Mestizen-Kultur Brasiliens durch eine Betonung schwarzer Identität in Frage zu stellen. Wer diesen Bereich anrührte, wie Gilberto Gil und Jorge Ben (vgl. Mello 2003: 320), riskierte den Ausschluss aus der MPB-Gemeinschaft. Daneben ist auf zwei Charakteristika der Festivaltexte aufmerksam zu machen: erstens waren oft Metasongs wie »Ponteio« oder Chico Buarques »A Banda« erfolgreich, die die Bedeutung von Musik innerhalb der Gesellschaft reflektieren (vgl. Dunn 1996: 115); $;^{12}$ zweitens besangen die Interpreten zwar vorwärtsgewandt und verhalten optimistisch, grundsätzlich aber unkonkret - und damit in gewisser Weise ähnlich eskapistisch wie die Jovem Guarda - den »dia que virá«, den erhofften besseren Tag in einer unbestimmten Zukunft (Galvão 1976). Allerdings waren konkretere Statements wegen der ständig drohenden Zensur im Grunde gar nicht möglich. Dies zeigt das Beispiel von Geraldo Vandrés »Caminhando《, ein offenes politisches Bekenntnis gegen die Militärregierung, das trotz massiven Drucks des Publikums keine Chance auf den Sieg beim 3. FIC 1968 hatte. Der Titel durfte zwar auf dem Festival präsentiert werden, wurde von der Zensur aber anschließend verboten. Außerdem musste Vandré das Land verlassen.

Auch hinsichtlich der Aufführung von Musik änderten die Festivals bestehende Gewohnheiten. Obwohl offiziell die beste Komposition in >reiner Form gesucht und prämiert werden sollte, war es den Juroren sowie dem Publikum natürlich nicht möglich, Aspekte der Performance in ihrer Bewertung auszublenden. Schon der Erfolg des Titels »Arrastão《 1965 beruhte zu einem Großteil auf dem Charisma und der Fernsehpräsenz der Interpretin Elis Regina, deren Lachen und Gesten wie für das Fernsehen gemacht schienen (Napolitano 2004: 55). Bei den Auftritten der Tropicalisten rückte die Performance dann in den Vordergrund und wuchs sich zum Happening aus. So wie Jimi Hendrix auf dem Monterey Festival seine Gitarre verbrannte (vgl. Mungen 2003), wurde im Fall von Caetano Veloso auch sein Auftritt beim 3. FIC 1968 zu einer vorbereiteten Aktion, deren Bedeutung erst durch den Einbezug der visuellen Präsenz von Kleidung, Gesten und Verhalten auf der Bühne lesbar wird. Veloso ersetzte den bis dahin obligatorischen Anzug

12 Auch Bossa-Nova-Stücke wie »Desafinado « und »Samba de uma Nota Só « sind Metasongs über die Bedeutung von Musik, allerdings ohne gesellschaftliche Komponente. 
mit Krawatte durch ein »proto-punk [...] outfit « (Stroud 2000: 103), ein futuristisches grün-silbernes Plastikkostüm. Hinzu kam, dass er die Präsentation seines Stückes »É proibido proibir« unvermittelt unterbrach und ein Gedicht von Fernando Pessoa rezitierte. Die Vorstellung wurde nochmals durch den Gastauftritt eines dem Publikum völlig unbekannten US-amerikanischen Hippies unterbrochen und endete mit einer aufgebrachten Rede Velosos gegen das Publikum und dem Aufruf an die Jury, inn gemeinsam mit Gilberto Gil zu disqualifizieren - eine Bitte, der die Jury allerdings nicht nachkam ist, denn »É proibido proibir« wurde zur Endrunde zugelassen. Veloso nahm daran aber aus eigenem Entschluss nicht teil (vgl. Mello 2003: 281).

\section{Tropicalismo}

Als avantgardistische Bewegung, die auf einem TV-Festival zum ersten Mal öffentlich in Erscheinung trat, hat der Tropicalismo eine besondere Bedeutung innerhalb der Geschichte der Festivals. Obwohl diese Bewegung von der bildenden Kunst ausging und sich bald auf Theater und Kino erstreckte, ${ }^{13}$ kam der populären Musik - und hier vor allem den Protagonisten Caetano Veloso und Gilberto Gil - in der öffentlichen Wahrnehmung und der Rezeption die tragende Rolle zu. Gemeinsamkeit des Zusammenschlusses von hauptsächlich bahianischen Musikern war einerseits die klischeehafte Betonung der marginalen Position Brasiliens, andererseits die plakative Zelebrierung der Konsumkultur, oft innerhalb eines Stückes kontrastiert mit Auswüchsen von Gewalt und sozialer Ungleichheit. Musikalisches Merkmal war die Verwendung von E-Gitarren und damit die - auch ideologische - Öffnung nach außen zur internationalen Popszene, zugleich aber die Revitalisierung traditioneller Instrumente wie dem Berimbau. Daneben erhielt erstmals in der Geschichte der populären Musik Brasiliens die Tonstudiotechnik mit ihren Möglichkeiten der Beeinflussung von Sound eine herausragende Rolle (Moehn 2000).

Die Entwicklung des Tropicalismo geht mit dem Verlauf der Festivals 1967-1968 einher. Auf dem 3. Festival da MPB setzten sich die Protagonisten Caetano Veloso und Gilberto Gil, zunächst noch unter der Genre-

13 Frederico Coelho (2002: 131) spricht ironisch von der »Heiligen Dreifaltigkeit des Tropicalismo « und nennt den Filmregisseur Glauber Rocha, den Theaterregisseur José Celso Martinez Corrêa sowie Caetano Veloso. Seine Analyse zeigt, dass neben diesen Hauptfiguren auch andere, exemplarisch bei inm der Journalist und Texter Torquato Neto, für das Zustandekommen und den Verlauf der tropicalistischen Bewegung bestimmend waren. 
bezeichnung Som universal, mit ihren Beiträgen durch. Der Einsatz von E-Gitarren kam einer Provokation gleich und spaltete das Publikum ähnlich wie Bob Dylans Auftritt in Newport 1965; dennoch erreichten sie den dritten bzw. fünften Platz. Im September 1968 gestaltete Veloso nach der Disqualifizierung von Gils »Questão da ordem « seinen Auftritt zum Happening, das vom Publikum gnadenlos ausgepfiffen wurde und so zum Eklat führte. Das 4. Festival da MBP bescherte den Tropicalisten im Dezember 1968 mit der Performance von Gal Costa und dem Siegertitel »São, São Paulo meu amor « von Tom Zé dann den Durchbruch, nun allerdings ohne die eigentlichen Anführer Gil und Veloso, die nach dem Inkrafttreten des fünften Institutionellen Akts (Al-5) ${ }^{14}$ der Militärregierung am 27. Dezember 1968 festgenommen wurden und im Jahr darauf ins Exil nach London gingen.

Für die Entwicklung der brasilianischen populären Musik und die Definition der MPB war der Tropicalismo entscheidend, denn er stellte einen >Protest< gegen die Protestmusik dar und sondierte das Feld zwischen den Positionen der engagierten Musiker und der Jovem Guarda neu. Damit verschob sich die Akzentuierung des Adjektivs »popular « im Kürzel MPB von der ursprünglichen brasilianischen Bedeutung auf die angloamerikanische: von der angestrebten Kommunikation mit der Arbeiterschicht auf den Einbezug der Konsumkultur (Dunn 2001: 68). Beachtenswert ist, dass dies im Rahmen der Festivals stattfand.

\section{Das Ende der Festivalära}

Bereits seit 1969 war die Aufmerksamkeit, die den Festivals in der Öffentlichkeit entgegengebracht wurde, rückläufig. Hauptursache hierfür war der Qualitätsverlust der Sendungen, der durch die verstärkte Einflussnahme der Zensur bei der Auswahl der Beiträge ausgelöst wurde. Die Stars vorangegangener Festivals befanden sich zudem im Exil. International hatte sich der Wettbewerb nicht durchgesetzt, wenn auch vereinzelt Neueinspielungen von Festivalstücken außerhalb Brasiliens zu Erfolgen wurden. ${ }^{15}$

Auch nach ihrem Ende 1972 hatten die Festivals einen massiven Einfluss auf die brasilianische Musiklandschaft. Da trotz eines sich ausbreitenden

14 Die Institutionellen Akte waren Verfassungszusätze, der Al-5 machte Verhaftungen und Ausweisungen in großem Umfang möglich und richtete sich u.a. auch gegen Studierende.

15 In der Bundesrepublik Deutschland z.B. »A Banda« in der Interpretation von France Gall (»Zwei Apfelsinen im Haar«) und - mit bescheidenerem Erfolg James Lasts Versionen von »Andança« (3. Platz, 3. FIC 1968) und »Cantiga por Luciana« (Sieger, 4. FIC 1969). 
Plattenmarktes keine neuen Talente hervorgebracht wurden, verzeichneten weiterhin die ehemaligen Wettbewerbstars hohe Verkaufszahlen (Napolitano 2002: 8). ${ }^{16}$ Erst in den 1980er Jahren wurde wieder eine neue Generation von Musikern erfolgreich.

Der Wettkampfgedanke hat, obwohl in Teilbereichen wie dem Karneval von Rio de Janeiro noch vorhanden, in der brasilianischen Musik seitdem spürbar an Bedeutung verloren (zu Wettbewerben zwischen 1979 und 2002 vgl. Stroud 2005). Dies kann als Beleg der These von Dietrich Helms (vgl. den Beitrag in diesem Band) gesehen werden, dass Wettbewerbe immer dann in Erscheinung treten, wenn das Funktionieren musikalischer Kommunikation gefährdet ist. Im Brasilien der 1960er Jahre wurden internationale musikalische Einflüsse von den Studierenden als Gefährdung für die Konstruktion einer populären Nationalkultur als Protest gegen die Militärregierung betrachtet. Die Musikfestivals sorgten durch die Verbannung von Rockmusikstücken der Jovem Guarda zumindest für eine begrenzte Zeit für eine gesicherte Kommunikation zwischen den studentischen Rezipienten und politisch engagierten Musikern. Aufschlussreich ist, dass bei der sich ab ca. 1985 formierenden Rockbewegung (Rock Brasileiro) Ressentiments gegen ausländische bzw. US-amerikanische Einflüsse nicht mehr vorhanden waren. Für deren Etablierung mit der Veranstaltung Rock in Rio war zwar wiederum ein Festival ausschlaggebend; der Wettkampfgedanke aber spielte keine Rolle mehr (Magaldi 1999: 310).

Mit dem Rückgang von musikalischen Wettbewerben geht seit einigen Jahren allerdings eine vermehrte Diskussion um die Festivalära 1965-1972 einher, die vor allem durch die Publikationen der Erinnerungen und Analysen von Zeitzeugen ausgelöst wurde: des ehemaligen Tonmeisters von TV Record Zuza Homem de Mello (2003), des Fernsehproduzenten Solano Ribeiro (2002), des Komponisten Nelson Motta (2000) und der Musiker Caetano Veloso (2002 [in Brasilien 1997]) und Tom Zé (2003). Im heutigen Brasilien beeindruckt offensichtlich wieder der Grad an gesellschaftlicher Bedeutung, den die musikalischen Wettbewerbe der späten 1960er und frühen 1970er Jahre besaßen. Auch wenn sie nicht zu einer Mobilisierung und weitergehenden politischen Aktivierung des Publikums geführt haben, boten die Festivals in einer Phase der politischen Unterdrückung einen Ort des Widerstandes. Heutzutage kommt brasilianischen Musikwettbewerben - wie

16 Und daneben die wenigen etablierten Musiker der 1960er Jahre, die mit den Festivals in keinem nennenswerten Zusammenhang standen, wie z.B. die BossaNova-Künstler der ersten Generation sowie Maria Bethânia, die zwar am 1. FIC teilnahm, dort aber nicht in Endrunde gelangte und sich danach vom Festivalbetrieb löste. 
zuletzt dem Festival da Música Brasileira 2000, dem von IBM organisierten Musikwettbewerb im Internet e-Festival und der nach dem >Superstar<Modell konzipierten Castingshow Fama - ähnlich wie in der Bundesrepublik Deutschland regelmäßig eine kurzzeitige Aufmerksamkeit zu, dauerhafte ästhetische oder gesellschaftliche Veränderungen auszulösen wird von ihnen aber nicht mehr erwartet.

\section{Literatur}

Araújo, Paulo Cesar de (2002). Eu não sou cachorro, não. Música popular cafona e ditatura militar. Rio de Janeiro: Record.

Campos, Augusto de (1993). Balanço da Bossa e outras bossas. São Paulo: Perspectiva (5. Aufl.; E: 1978).

Coelho, Frederico (2002). »A formação de um tropicalista: um breve estudo da coluna >Música Popular<, de Torquato Neto. «In: Estudos Históricos 30, S. 129-146; http://cpdoc.fgv.br/revista/arq/335.pdf (Zugriff: 27.1.2005).

Dunn, Christopher (1996). The Relics of Brazil: Modernity and Nationality in the Tropicalista Movement. Ph.D. Dissertation, Brown University Providence, RI.

Dunn, Christopher (2001). Brutality Garden. Tropicália and the Emergence of a Brazilian Counterculture. Chapel Hill: University of North Carolina Press.

Galvão, Walnice Nogueria (1976). Saco de Gatos. Ensaios Críticos. São Paulo: Duas Cidades.

Godi, Antonio J. V. dos Santos (2002). »Reggae and Samba-Reggae in Bahia: A Case of Long-Distance Belonging. «In: Brazilian Popular Music \& Globalization. Hg. v. Charles A. Perrone und Christopher Dunn. New York: Routledge, S. 207-219.

Magaldi, Christina (1999). »Adopting Imports: New Images and Alliances in Brazilian Popular Music of the 1990s. «In: Popular Music 18, Nr. 3, S. 309-329.

McCann, Bryan (2004). Hello, Hello Brazil. Popular Music in the Making of Modern Brazil. Durham/London: Duke University Press.

Mello, Zuza Homem de (2003). A Era dos festivais. Uma parábola. São Paulo: Ed. 34.

Moehn, Frederick (2000). »In the Tropical Studio: MPB Production in Transition. «In: Studies in Latin American Popular Culture 19, S. 57-66.

Motta, Nelson (2000). Noites tropicais. Improvisos e memórias musicais. Rio de Janeiro: Objetiva.

Mungen, Anno (2003). »The Music is the Message: The Day Jimi Hendrix Burnt his Guitar.« In: Popular Music and Film. Hg v. Ian Inglis. London: Wallflower, S. 6076.

Napolitano, Marcos (2001). s Seguindo a canção: engajamento politico e indústria cultural na MPB (1959-1969). São Paulo: Annablume.

Napolitano, Marcos (2002). »A música popular brasileira (MPB) dos anos 70: resistência política e consumo cultural.«In: Actas del IV Congresso Latinoamericano de la Asociación Internacional para el Estudio de la Música Popular; http://www.hist.puc.cl/historia/iaspmla.html (Zugriff: 27.1.2005).

Napolitano, Marcos (2004). Cultura brasileira: utopia e massificação (1950-1980). São Paulo: Contexto.

Naves, Santuza Cambraia (2001). Da Bossa Nova à Tropicália. Rio de Janeiro: Jorge Zahar. 
Perrone, Charles A. (2002). »Myth, Melopeia, and Mimesis: Black Orpheus, Orfeu, and Internationalization in Brazilian Popular Music. «In: Brazilian Popular Music \& Globalization. Hg. v. Charles A. Perrone und Christopher Dunn. New York, London: Routledge, S. 46-71.

Pinto, Tiago de Oliveira (1995). »Forró in Brasilien, Musik für Dienstmädchen und Taxifahrer? In: PopScriptum 3, S. 52-79; http://www2.hu-berlin.de/fpm/ popscrip/themen/pst03/pst03040.htm (Zugriff: 27.1.2005).

Ribeiro, Solano (2002). Prepare seu coração. A história dos grandes festivais. São Paulo: Geração Editorial.

Schreiner, Claus (1977). Música Popular Brasileira. Darmstadt: Tropical Music.

Stroud, Sean (2000). »>Música é para o povo cantar<: Culture, Politics, and Brazilian Song Festivals, 1965-1972.« In: Latin American Music Review 21, Nr. 2, S. 87117.

Stroud, Sean (2005). Disco é Cultura: MPB and the defence of tradition in Brazilian popular music. Ph.D. Dissertation., King's College, University of London.

Travassos, Elizabeth (2000). »Ethics in the Sung Duels of North-Eastern Brazil: Collective Memory and Contemporary Practice. « In: British Journal of Ethnomusicology 9, Nr. 1 (Special issue: Brazilian Musics, Brazilian Identities, hg. v. Suzel Ana Reily), S. 61-94.

Treece, David (1997). "Guns and Roses. Bossa Nova and Brazilian's Music of Popular Protest, 1958-68.«In: Popular Music 16, S. 1-29.

Veloso, Caetano (2002). Tropical Truth. New York: Knopf.

Vilarino, Ramon Casas (2000). A MPB em movimento: música, festivais e censura. São Paulo: Olho d'Água.

Villaça, Mariana Martins (2004). Polifonia tropical: experimentalismo e engajamento na música popular (Brasil e Cuba, 1967-1972). São Paulo: Humanitas/ $\mathrm{FFLCH} / \mathrm{USP}$.

Zan, José Roberto (2003). »Popular Music and Policing in Brazil.«In: Policing Pop. Hg. v. Martin Cloonan und Reebee Garofalo. Philadelphia: Temple University Press, S. 205-220.

Zé, Tom (2003). Tropicalista lenta luta. São Paulo: Publifolha.

Zoller, Rüdiger (2000). »Präsidenten - Diktatoren - Erlöser: Das lange 20. Jahrhundert. «In: Walther L. Bernecker, Horst Pietschmann und Rüdiger Zoller, Eine kleine Geschichte Brasiliens. Frankfurt/M.: Suhrkamp, S. 215-320.

\begin{abstract}
Music contests have a long tradition in Brazil as the carnival in Rio de Janeiro shows. The phenomenon of music contests was most conspicuous between 1965 and 1972 when many TV-stations organised and broadcast a number of so called festivals that had a widespread impact on the development of popular music in Brazil. A large number of musicians which are still regarded as the most important ones in Brazil performed on these festivals for the first time. This paper focuses on the role of TV festivals as a platform for a left-wing orientated student movement in search of a national-popular culture as a means of protest against the military regime and on the musical innovations which arose in the context of the festivals.
\end{abstract}

